\title{
The wind-wind collision hole in eta Car
}

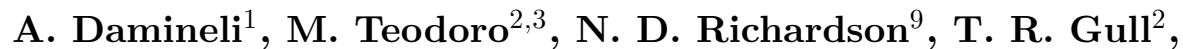 \\ M. F. Corcoran ${ }^{2,3}$, K. Hamaguchi ${ }^{2,3}$, J. H. Groh ${ }^{5}$, G. Weigelt ${ }^{6}$, \\ D. J. Hillier ${ }^{7}$, C. Russell ${ }^{2}$, A. Moffat ${ }^{4}$, K. R. Pollard ${ }^{8}$ and \\ T. I. Madura ${ }^{10}$
}

${ }^{1}$ Inst. de Astron., Geofísica e Ciências Atmosféricas, Univ. de São Paulo, R. do Matão 1226, São Paulo 05508-900, Brazil

email: augusto.damineli@iag.usp.br

${ }^{2}$ Astroph. Sci. Division, Code 660, NASA Goddard Space Flight Center, Greenbelt, MD 20771, USA

${ }^{3}$ USRA, 7178 Columbia, MD 20146, USA

${ }^{4}$ Départ. de Physique, Univ. de Montréal, CP 6128, Succursale: Centre-Ville, Montréal, QC, H3C 3J7, Canada

${ }^{5}$ School of Physiscs, Trinity College Dublin, The Un. of Dublin, Dublin 2, Ireland

${ }^{6}$ Max-Planck-Institut for Radioastronomy, Auf dem Hügel 69, D-53121 Bonn, Germany

${ }^{7}$ Depart. of Phys. and Astr., Univ. of Pittsburgh, 3941 O'Hara Street, Pittsburgh, PA 15260, USA

${ }^{8}$ Department of Physics and Astronomy, University of Canterbury, Chirstchurch, New Zealand

${ }^{9}$ Ritter Observ., Depart. of Phys. and Astr., The University of Toledo, Toledo, OH 43606-3390, USA

${ }^{10}$ San Jose State University, Depart. of Physics and Astronomy, San Jose, CA, USA

\begin{abstract}
Eta Carinae is one of the most massive observable binaries. Yet determination of its orbital and physical parameters is hampered by obscuring winds. However the effects of the strong, colliding winds changes with phase due to the high orbital eccentricity. We wanted to improve measures of the orbital parameters and to determine the mechanisms that produce the relatively brief, phase-locked minimum as detected throughout the electromagnetic spectrum. We conducted intense monitoring of the He II $\lambda 4686$ line in $\eta$ Carinae for 10 months in the year 2014, gathering $\sim 300$ high S/N spectra with ground- and space-based telescopes. We also used published spectra at the FOS4 SE polar region of the Homunculus, which views the minimum from a different direction. We used a model in which the He II $\lambda 4686$ emission is produced by two mechanisms: a) one linked to the intensity of the wind-wind collision which occurs along the whole orbit and is proportional to the inverse square of the separation between the companion stars; and b) the other produced by the 'bore hole' effect which occurs at phases across the periastron passage. The opacity (computed from 3D SPH simulations) as convolved with the emission reproduces the behavior of equivalent widths both for direct and reflected light. Our main results are: a) a demonstration that the He II $\lambda 4686$ light curve is exquisitely repeatable from cycle to cycle, contrary to previous claims for large changes; b) an accurate determination of the longitude of periastron, indicating that the secondary star is 'behind' the primary at periastron, a dispute extended over the past decade; c) a determination of the time of periastron passage, at $\sim 4$ days after the onset of the deep light curve minimum; and d) show that the minimum is simultaneous for observers at different lines of sight, indicating that it is not caused by an eclipse of the secondary star, but rather by the immersion of the wind-wind collision interior to the inner wind of the primary.
\end{abstract}

Keywords. stars: individual ( $\eta$ Carinae $)$ - stars: massive — binaries: general — stars: circumstellar matter — atomic data — atomic processes — radiation mechanisms: general — plasmas 


\section{Introduction}

Eta Carinae is one of the most luminous evolved stars in the local Universe (Davidson \& Humphreys 1997). It became famous after the great eruption in the 1840's, which created the beautiful bipolar Homunculus flow and there were additional smaller mass ejections. The discovery that it is long period eccentric binary (Damineli 1996, Damineli, Conti \& Lopes 1997) provided the potential to determine the mass of the companions and to explain a number of complex, variable features. One very interesting is the connection between the sharp photometric peaks during the great eruption and timing of periastron passages, indicating that at periastron, there is strong interaction between the companion stars (Damineli 1996, Prieto et al. 2014). Moreover, the shape of the homunculus displays features formed when the ejecta was a thousand times smaller than at the time of the eruption (Steffen et al. 2014).

In practice, variations of the system do not easily lead to the physical parameters: both companions have strong winds, with especially the primary's known to be strongly variable, leading to strong and variable wind-wind collision (WWC), which give rise to many complex and variable phenomena like free-free and free-bound emission, radio emission, high and low energy emission lines. A recent discovery (Steiner \& Damineli 2004) of a "burst" in the He II $\lambda 4686$ line, because of its sharp and phase-locked behaviour (Teodoro et al. 2012), turned out to be a key phenomenon to study the binary system. As shown by Madura \& Owocki (2010) and Madura et al. (2013), the 'bore hole' effect can account for the escaping of UV radiation from the inner interacting binary to the external regions, through the cavity (filled by the sparser secondary wind) produced by the WWC. 3D SPH simulations are necessary to calculate the opacity to each direction. Such a tool is critical to explore the nature of the 'low excitation events'. As shown by Ishibashi et al. (1999), the minimum in the X-ray light curve is too long to be explained by an eclipse, even when adding a disk around the primary star. The radio light-curve also shows that the minimum is not restricted to our direction (Duncan \& White 2003). The radio nebula grows and shrinks continuously, reaching a minimum simultaneously with all other features. It could be that an eclipse is possible involved in the minimum, but it is not the main or the sole mechanism.

Results in this paper are described in details by Teodoro et al. (2016).

\section{The 2014.6 monitoring campaign of the He II $\lambda 4686$ line in $\eta$ Carinae}

Observations of the He II $4686 \AA$ emission were done with HST/STIS beginning after the periastron event in 2009 through 2015 for a total of twenty one intervals (Gull et al. 2016, Mehner et al. 2015). Even more observations with ground-based telescopes were carried out during 2014, with 114 spectra from CTIO/Chiron, 90 spectra from OPD/LNA, 37 from SOAR/Goodman, 26 from MJUO/Hercules and 19 from CASLEO/REOSC. The group of amateurs SASER (Southern Astro Spectroscopy Email Ring) contributed 46 spectra. We also used 9 spectra from the Hexapod Telescope/BESO collected in critical times after the 2009.1 periastron. The resolution of the spectra varied among the facilities employed, but $\mathrm{S} / \mathrm{N}$ was $>200$ per resolving element for the vast majority of the data. The typical uncertainty of the EW (equivalent width) measurements was $\sim 2 \AA$ when inter-comparing contemporary data from different observers. For such a faint line, in a rich emission line spectrum, the placement of the continuum is challenging. Although it is impossible to obtain an unbiased measurement of the line intensity for an isolated spectrum, the variability can be derived in a robust way by a careful rectification of the 
stellar continuum (using a flux standard star) and using always the same wavelength windows (blue: $4600 \pm 5 \AA$; red: $4742 \pm 1 \AA$ ) and line integration (4675-4694 $\AA$ ). Results are insensitive to instrumental setup for ground-based telescopes, for which the slit samples a region of 1-3 $\AA$ projected on the sky. The HST/STIS spectra, with spatial sampling was $\sim 0.1$ arcsec, excludes considerable extended wind and nebular structures, which contribute considerable continuum, thus decreasing the equivalent width of the $4686 \AA$ feature. Fortunately thirteen of the HST/STIS observations were mapped across the $2 \times 2$ $\operatorname{arcsec}^{2}$ region centered on eta Car. Comparison of the integrated mapped spectra were found to be compatible with measures of the ground-based spectra Teodoro et al. 2016).

We used several different procedures to derive a period that better fits the light-curve using the He II $\lambda 4686$ line and also other features measured by different authors, like broad-band photometry, X-rays and radio flux, obtaining $\mathrm{P}=2022.7 \pm 0.3 \mathrm{~d}$ Damineli et al. (2008). It is important to notice that such a high accuracy (1 part in 10000 ) opens the possibility to detect changes in the period due to the mass loss in future periastron passages.

The EW He II $\lambda 4686$ light-curve resembles that of X-rays. It stays at a fairly constant level $(\sim 50 \mathrm{~mA})$ for about 4.5 years up to phase=0.9. The He II EW slowly rises for $\sim 4$ months, then rapidly grows for 2 months, reaching a peak of $\sim 3.5 \AA$. The fading phase reaches zero intensity in a timescale as short as 2 weeks. Different from X-rays, it stays at minimum for just $\sim 1$ week and recovers with phase-locked behaviour, to reach another local peak (P3) $\sim 30$ days after the minimum. The light curve then returns to the low level of $\sim 50 \mathrm{~m} \AA$ at $\sim 2$ months after the minimum. The similarity between the X-ray and He II $\lambda 4686$ light curves when approaching the minimum and their large differences after that indicate that different mechanisms are controlling these two light curves in the recovery phase.

\section{The light curve minimum seen from two directions}

Comparison of He II $\lambda 4686$ as seen from different view points provides information on the periastron and symmetry, which is tilted out of the sky plane at 45 deg latitude (Gull et al., 2009, Madura et al. 2013). Our vantage point views the orbital axis at $45 \mathrm{deg}$ latitude. The FOS4 position reflects light from the orbital polar region - 90 deg latitude (Mehner et al. 2015). With a time-delay of 17 days, derived from the Homunculus shape and expansion rate (Smith 2006, both light curves show coincident temporal events. Once this correction is done, the time of the minimum of both light curves are in close coincidence in time. The immediate conclusion is that the 'event' cannot be an eclipse, as otherwise already hinted by the radio light curve. The simple interpretation is that the 'event' is produced by the blanketing of the UV ionizing radiation when the secondary star penetrates the inner dense wind of the primary. The cavity inside the WWC shock cone is wrapped by the orbital motion to the point that it no longer enables the radiation emitted at its apex to escape in any direction. This happens when the secondary star reaches periastron so that this critical point can be determined from the combination between the direct and reflected light curves.

The orbital orientation (specially the longitude of periastron) can be derived from modelling the He II $\lambda 4686$ light curve. We used a model in which the intensity of emission goes as $1 / D^{2}$ (D is the distance between the companion stars), following Fahed et al. (2011). Since the light curve grows/fades at a steeper pace around periastron, there must be an additional source which is present just when the apex of the WWC is at $\sim 1$ month from periastron. We assume that such emission comes from recombination of $\mathrm{He}^{2+}$ ionized from $\mathrm{He}^{+}$by the UV and soft X-rays from the WWC apex. This demands 


\section{The "bore hole" effect}

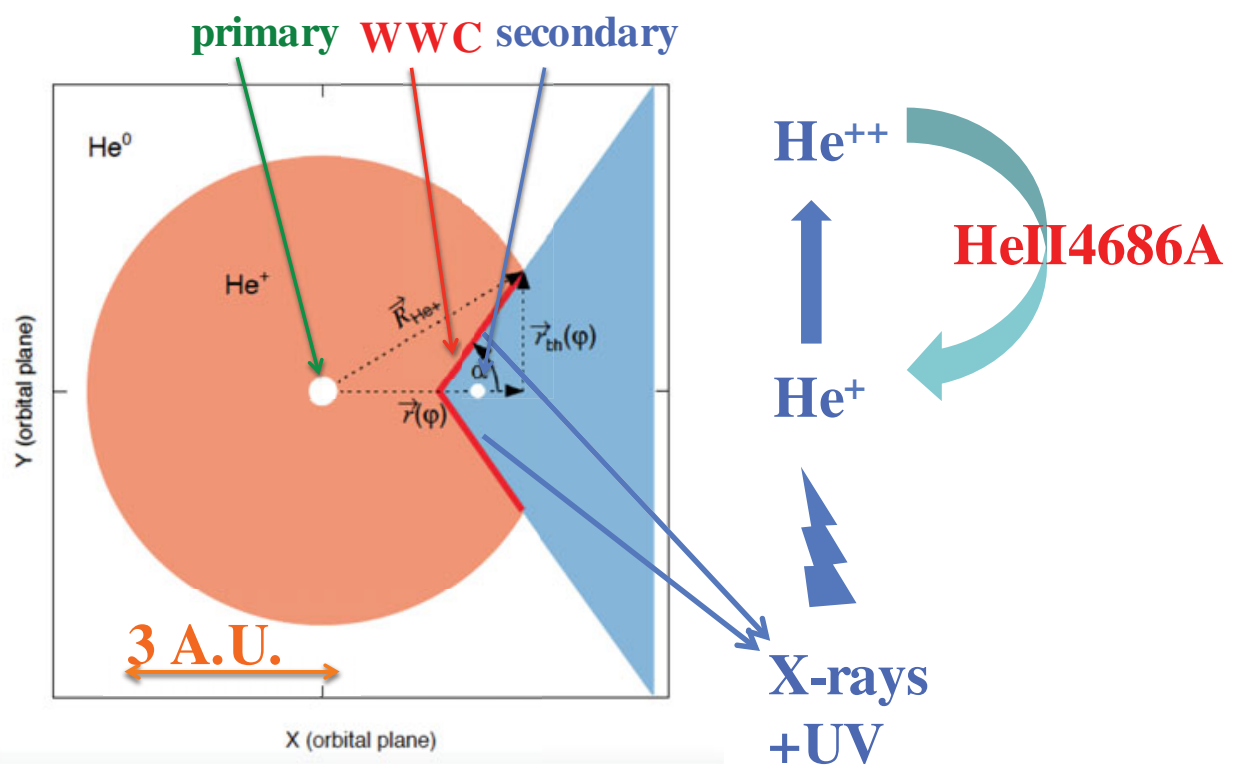

Figure 1. The bore hole effect, following Madura \& Owocki (2010). When the WWC apex gets close and enters inside the $\mathrm{He}^{+}$sphere, it is illuminated by a powerful extreme-UV radiation source and is ionized to $\mathrm{He}^{2+}$. Recombination to $\mathrm{He}^{+}$produces He II $\lambda 4686$ photons. This accounts for the steep increase of the He II $\lambda 4686$ light-curve for about \pm 1 month on either side of periastron. This emission adds up to the $1 / D^{2}$ effect that modulates the He II $\lambda 4686$ emission along the entire orbit as the distance (D) between the companion stars changes.

a huge reservoir of $\mathrm{He}^{+}$, as is predicted by Hillier et al. (2011) model for the atmosphere of the primary star. When the WWC apex penetrates that sphere $(\mathrm{R}=3 \mathrm{AU})$ a luminous source of He II $\lambda 4686$ is ignited and grows vey fast as the star approaches periastron. Both sources of emission add up and peak symmetrically around periastron. Electron scattering in the primary's wind produces opacity and the visibility of the He II $\lambda 4686$ source gets modulated according to the orbital orientation relative to the observer. In addition to the primary star wind, the cavity inside the WWC shock cone also impacts the opacity. This is the 'bore hole' mechanism (Madura and Owocki 2010 ). We used 3D $\mathrm{SPH}$ simulations to model the He II II $\lambda 4686$ light curve from the direct and reflected views. A very narrow range of periastron longitudes ( $\omega=234 \mathrm{deg}-252 \mathrm{deg})$ were allowed and the orbital inclination also could be constrained. It is interesting to see how good the fit of both light curves was. Even minor details, like the smaller amplitude of the light curve in reflected light, was well reproduced.

It is also noticeable that the time of periastron passage derived from this model is in close agreement with the time of minimum for hard X-rays. The fact that the recovery from the minimum in $\mathrm{X}$-rays does not repeat from cycle to cycle, indicates that there additional mechanisms at work (Corcoran et al. 2010). For example, some X-ray emission is produced far from the WWC apex or it may be subject to density fluctuations due to clumping in the primary's wind. 


\section{Conclusions}

Periodicity. The period length defined by the He II $\lambda 4686$ line during the last 3 cycles is

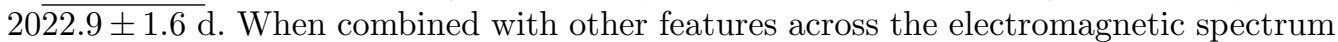
(Damineli et al. 2008) it is better constrained to $\mathrm{P}=2022.7 \pm 0.3 \mathrm{~d}$.

Time of periastron passage. The periastron passage occurs $\sim 4$ days after the He II $\lambda 4686$ line reaches its minimum $(\mathrm{Eq} \mathrm{W} \sim 0)$ at $\mathrm{JD}=2,456,874.1 \pm 2.3$ days.

Stability of the HeII $\lambda 4686$ light-curve. There are no statistically significant changes in the line intensities when comparing the 2009.1 and the 2014.6 events contrary to previous claims (Mehner et al. 2015). The P2 peak could have increased by up to $\sim 30$ percent in the 2014.6 periastron as compared to the previous one.

Ingredients to reproduce the He II $\lambda 4686$ light curve. The light curve is well reproduced by adopting for the emission a 1/D $\mathrm{D}^{2}$ law like in Fahed et al. (2011) along the whole orbit of WR140, plus a "bore hole" component like in Madura \& Owocki (2010) which works when the WWC is very close to or inside the $\mathrm{He}^{+}$sphere around the primary star. The absorption is calculated by $3 \mathrm{D} \mathrm{SPH}$ simulations.

The orbital parameters. The simultaneous fit to direct and reflected light curves gives the longitude of periastron as $\omega=234-252 \mathrm{deg}$, in such a manner that the secondary star is "behind" the primary at periastron. The orbital inclination angle is $i=135-153 \mathrm{deg}$. The orbital eccentricity was adopted as $e=0.9$.

Verification from high spatial resolution. These orbital parameters are in excellent agreement with high spatial resolution observations reported by Gull et al. (2016) with HST (50 mas resolution) showing the high excitation lines at the opening of the WWC cavity. Weigelt et al. (2016) used the AMBER camera at the ESO/VLTI (6 mas resolution) to show that the WWC cavity is opened in our direction along most of the orbit, also in agreement with our orbital orientation.

\section{References}

Corcoran M.F., Hamaguchi, K., Pittard, J.M. et al. 2010 ApJ, 725, 1528

Damineli A., 1996, ApJ (Letters), 460, L49

Damineli A., Conti P. S., Lopes D. F., 1997, New Astron., 2, 107

Damineli A., et al. 2008, MNRAS, 384, 1649

Davidson K., Humphreys R. M., 1997, ARAA, 35, 1

Duncan R.A \& Whithe, S.M. 2003 2003, MNRAS, 338, 425

Fahed R., et al., 2011, MNRAS, 418, 2

Gull T., et al., 2009, MNRAS, 396,1308

Gull T., et al., 2016, MNRAS, 462, 3196

Hillier D. J., Davidson K., Ishibashi K., Gull T., 2001, ApJ, 553, 837

Ishibashi, K., Corcoran, M. F., et al. 1999,ApJ, 524, 983

Madura T. I., Owocki S. P. 2010,RMAA, 38, 52

Madura T. I., et al., 2013, MNRAS, 436, 3820

Mehner A., et al., 2015, A\&A, 578, 122

Prieto et al., 2014 ApJ, 787, L8

Smith N., 2006, AJ, 644, 1151

Steffen W., et al., 2014, MNRAS, 442, 3316

Steiner J. E., Damineli A., 2004, ApJ (Letters), 612, L133

Teodoro M., et al., 2012, ApJ, 746, 73

Teodoro M., Damineli, A., et al. 2016, ApJ, 819, 131

Weigelt G., et al., 2016, A\&A, 594, 106 